\title{
Personalia
}

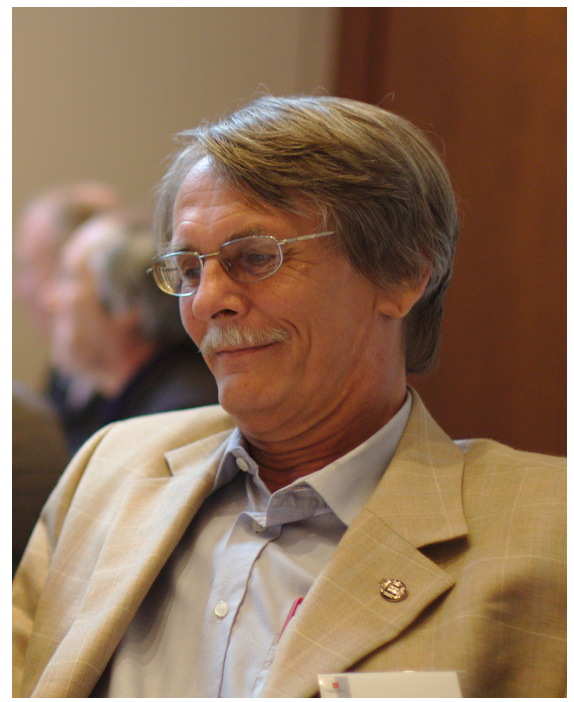

\section{Wolfhard Janke turns 60}

On December 11th, 2015, Wolfhard Janke, a member of the Editorial Board of "Condensed Matter Physics” and a leading expert in computer simulations in condensed matter physics, celebrated his 60th birthday.

Wolfhard was born and grew up in West Berlin. He studied physics and mathematics at the Freie Universität Berlin, where he received his PhD working under the guidance of Hagen Kleinert in 1985. He was awarded the Joachim Tiburtius Prize of the City of Berlin for the best doctoral dissertation in 1986. He stayed at this university for his habilitation degree, in time to see the Berlin Wall come down and Germany reunited in 1989/1990. After interludes at Florida State University in Tallahassee and Forschungszentrum Jülich in 1991/1992, he won a prestigious Heisenberg Fellowship of the Deutsche Forschungsgemeinschaft (DFG) that brought him to Kurt Binder's group at Johannes GutenbergUniversität Mainz, where he worked from 1992 until 1997. In 1998, he was appointed to a Chair in Theoretical Physics at Universität Leipzig. There, apart from establishing an extremely productive and still growing research group in statistical physics, he has served in a number of senior positions, including that of Vice Dean of the Faculty of Physics and Earth Sciences as well as the directorship of the Institute for Theoretical Physics and that of the Centre for Theoretical Sciences. He was also appointed to a number of external faculty positions, most recently in 2015 as Adjunct Professor of the Center for Simulational Physics at the University of Georgia, USA.

His oeuvre is remarkable not only for its variety, but also its sheer size: he has over 400 scientific publications to date, with subjects ranging through quantum gravity, the $X Y$ model, biopolymers and even the modelling of football scores. Wolfhard's research started in field theory in Kleinert's group in Berlin, but he soon turned his attention to studies of condensed-matter systems by means of computer simulations. Nonetheless, his background in field theory and high-energy physics has influenced his research topics, the rigour of his methods and his perspective to the present day. Wolfhard's accuracy and meticulous attention to detail are legendary and form a cornerstone of his excellent reputation in the community.

The leitmotif for most of his work has been the study of phase transitions and critical phenomena, although he has also made important contributions to the development of discrete approaches to quantum gravity, studying the Regge and dynamical triangulations approaches. He has determined precise critical exponents for a large range of systems, studied logarithmic scaling corrections, percolation and 
self-avoiding walks, the nucleation behaviour of the ordered phase and the effect of quenched disorder on systems undergoing phase transitions, including the spin-glass problem, to name but a few examples. Another important focus of his prolific research activity has been the study of systems undergoing firstorder phase transitions. This ranges from one of his first papers, written during his PhD studies, dealing with 2D disclination melting, through the study of interface tensions with the multicanonical method, to some of his most recent works on modified scaling in discontinuous transitions in the presence of degeneracies.

While much of his work until the early 2000s dealt with spin systems, in the last 10 years his focus has shifted towards soft-matter problems such as structural transitions in polymer systems. The very same imaginative use of numerical methods and rigorous analysis he showed in the studies of spin systems has also been applied to simulations of coarse-grained models for polymers and proteins. In an extensive series of works, he investigated adsorption phenomena of polymers at surfaces and the confinement of macromolecules in different geometries.

An underlying theme of much of his research is the advancement of the methodology for Monte Carlo simulations in statistical and soft-matter physics. He was one of the first researchers to apply cluster updates to perform high-precision studies of the critical behaviour of $X Y$ and Heisenberg models. He developed and used variants of the multicanonical method to study many different systems undergoing first-order transitions and also models with complex free-energy landscapes, where conventional techniques fail. Wolfhard also proposed refinements to many other numerical techniques, including parallel tempering and the statistical analysis of simulation data. He is widely regarded as one of the leading practitioners in the field.

Wolfhard is very active in research training and dissemination. He has supervised more than $40 \mathrm{PhDs}$, Diploma and MSc graduates. He has also been a tireless organiser of conferences and workshops, including the Spring Meeting of the German Physical Society (2002) and the annual meeting of the Middle European Cooperation in Statistical Physics (2009). His most well-known organisational activity, that has become something of an institution in its own right, is the CompPhys series of meetings held at the end of November every year in Leipzig. Not only has this series of events, which was held for the 16th time this year, turned out to be a powerhouse of new and existing collaborations, but it has also proved to be an extremely popular social occasion. It forms a non-negotiable recurring item in the calendars of many statistical physicists.

Above all, however, we cherish Wolfhard for his deep insights that he is always happy to share in discussions and his sociable character that has been the nucleus for the many collaborations he has initiated over the years. These have resulted in a number of longtime partnerships, including international network projects such as a sequence of RTN projects on Random Geometries with partners all over Europe, and the International Doctoral College for the Statistical Physics of Complex Systems, Leipzig-LorraineLviv-Coventry $\left(\mathbb{L}^{4}\right)$.

The CMP Editorial board, on behalf of numerous colleagues and friends around the world, thanks Wolfhard Janke for his important contributions to our field, warmly congratulates him on the occasion of his jubilee and wishes him many further successes on his way. 